\title{
The effects of surgical treatment and sclerotherapy for intramuscular venous malformations: a comparative clinical study
}

\author{
Yun Hyun Kim ${ }^{1}$, Jeong Yeop Ryu ${ }^{1}$, Joon Seok Lee ${ }^{1}$, Seok Jong Lee, ${ }^{2,3}$, Jong Min Lee ${ }^{3,4}$, \\ Sang Yub Lee ${ }^{3,4}$, Seung Huh ${ }^{3,5}$, Ji Yoon $\mathrm{Kim}^{3,6}$, Ho Yun Chung ${ }^{1,3,7,8}$ \\ Departments of ${ }^{1}$ Plastic and Reconstructive Surgery and ${ }^{2}$ Dermatology, School of Medicine, Kyungpook National University, Daegu; ${ }^{3}$ Vascular \\ Anomalies Center, Kyungpook National University Hospital, Daegu; Departments of ${ }^{4}$ Radiology, ${ }^{5}$ Surgery, and ${ }^{6}$ Pediatrics, School of \\ Medicine, Kyungpook National University, Daegu; ${ }^{7}$ Cell and Matrix Research Institute and ${ }^{8} B K 21$ FOUR KNU Convergence Educational \\ Program of Biomedical Sciences for Creative Future Talents, School of Medicine, Kyungpook National University, Daegu, Korea
}

\begin{abstract}
Background Venous malformations (VMs) are the most common type of vascular malformations. Intramuscular venous malformations (IMVMs) are lesions involving the muscles, excluding intramuscular hemangiomas. The purpose of this study was to compare clinical outcomes between patients with IMVMs who were treated with sclerotherapy and those who were treated with surgical excision.

Methods Of 492 patients with VMs treated between July 2011 and August 2020 at a single medical center for vascular anomalies, 63 patients diagnosed with IMVM were retrospectively reviewed. Pain, movement limitations, swelling, and quality of life ( $\mathrm{OOL}$ ) were evaluated subjectively, while radiological outcomes were assessed by qualified radiologists at the center. Complication rates were also evaluated, and radiological and clinical examinations were used to determine which treatment group (sclerotherapy or surgical excision) exhibited greater improvement.

Results Although there were no significant differences in pain $(P=0.471)$, swelling $(P=0.322)$, or the occurrence of complications $(P=0.206)$ between the two treatment groups, the surgical treatment group exhibited significantly better outcomes with regard to movement limitations $(P=0.010), Q 0 L(P=0.013)$, and radiological outcomes $(P=0.017)$. Moreover, both duplex ultrasonography and magnetic resonance imaging showed greater improvements in clinical outcomes in the surgical excision group than in the sclerotherapy group.

Conclusions Although several studies have examined IMVM treatment methods, no clear guidelines for treatment selection have been developed. Based on the results of this study, surgical excision is strongly encouraged for the treatment of IMVMs.
\end{abstract}

Keywords Intramuscular venous malformation / Surgery / Sclerotherapy

\author{
Correspondence: Ho Yun Chung \\ Department of Plastic and \\ Reconstructive Surgery, School of \\ Medicine, Kyungpook National \\ University, 130 Dongdeok-ro, Jung- \\ gu, Daegu 41944, Korea \\ Tel: +82-53-420-5688. \\ Fax: +82-53-425-3879. \\ E-mail: chunghoyun@gmail.com
}

This work was supported by a National Research Foundation of Korea (NRF) grant, funded by the Korean government (MSIT) (No. 2020R1A2C2009496).

\section{INTRODUCTION}

Venous malformations (VMs), the most common type of vas- cular malformations, are a consequence of abnormal angiogenic development, although the specific mechanisms involved are still unclear. VMs may occur in various parts of the body, with

Copyright $\odot 2021$ The Korean Society of Plastic and Reconstructive Surgeons

This is an Open Access article distributed under the terms of the Creative Commons Attribution Non-Commercial License (https://creativecommons.org/

licenses/by-nc/4.0/) which permits unrestricted non-commercial use, distribution, and reproduction in any medium, provided the original work is properly cited. I www.e-aps.org 
approximately $40 \%$ of lesions occurring in the head and neck region. These lesions do not disappear naturally and typically present as non-pulsating soft masses accompanied by black and blue skin discoloration and pain. Moreover, the size of the lesion and associated pain may increase with hormonal changes such as puberty, menstruation, and pregnancy. VM may also be associated with swelling, limitation of movement, and discomfort, leading patients to seek treatment. Conservative treatment methods include the use of compression garments, sclerotherapy, cryoablation, and surgical resection. The choice of treatment is based on the size and location of the lesion, associated symptoms, and the presence of neural involvement. However, there are no clear guidelines for the selection of treatment options [1].

The International Society for the Study of Vascular Anomalies classifies intramuscular venous malformations (IMVMs) as a common type of VMs, distinct from intramuscular hemangio$\mathrm{ma}$, which is a provisionally unclassified vascular anomaly [2]. For this study, IMVMs were defined as common VMs limited to the muscle, excluding intramuscular hemangioma.

IMVMs are relatively rare and are typically associated with symptoms such as severe pain, swelling, and functional limitations [2-6]. The low incidence of IMVM and variation in the location of lesions make it difficult to compare the outcomes of various treatment methods, the most common of which are sclerotherapy and surgery [7]. The choice of treatment is typically based on the clinical characteristics of the patient, the patient's treatment preferences, and the results of imaging examinations.

Therefore, the purpose of this study was to compare the clinical outcomes of sclerotherapy and surgical excision used to treat IMVMs, with the aim of developing a treatment guideline for VMs and informing treatment protocols for IMVMs.

\section{METHODS}

\section{Patients and data}

The Institutional Review Board of Kyungpook National University Hospital (IRB No. 2018-10-010) approved the study. Of 492 patients diagnosed with VM and treated between July 2011 and August 2020 at the Vascular Anomalies Center, 63 patients who presented with IMVMs were retrospectively reviewed.

Patients who underwent both sclerotherapy and excision were excluded from the study. Only patients who underwent sclerotherapy or excision alone were included in the study, and the study was further limited to those who underwent sclerotherapy or excision once. Patients' medical history, demographics, presenting symptoms, lesion characteristics (location, size, and extent of muscle involvement), and clinical outcomes were re- corded. Limitation of movement, swelling, and quality of life (QOL) were subjectively assessed, and the radiological examination was carried out by a designated radiologist at the hospital. Complications included skin necrosis, venous thrombosis, and nerve injury. The shortest follow-up period was 10 months and the longest follow-up period was 5 years.

\section{Diagnostic imaging}

The diagnostic protocol included duplex ultrasonography (DUS) and magnetic resonance imaging (MRI), which were used to grade and categorize the IMVM lesions. IMVM grading was carried out as follows: (1) grade 1, well-defined lesions $\leq 5$ $\mathrm{cm}$; (2) grade $2 \mathrm{~A}$, well-defined lesions $>5 \mathrm{~cm}$; (3) grade $2 \mathrm{~B}$, illdefined lesions $\leq 5 \mathrm{~cm}$; and (4) grade 3, ill-defined lesions $>5$ $\mathrm{cm}$ [8]. Based on MRI results, the patients were further categorized into two groups, those with single muscle involvement and those with IMVM invasion into multiple muscles.

\section{Treatment}

The patients were treated using either surgery or sclerotherapy, taking the patient's age, location of the lesion, grade of the lesion, symptoms, and cosmetic factors into consideration. Sclerotherapy was performed by injecting bleomycin and/or Fibrovein (sodium tetradecyl sulfate concentrate) 3\% solution intraluminally and/or intralesionally by a designated radiologist. Surgical excision included complete removal of the lesion where possible, although remnant lesions were present in some cases.

\section{Clinical outcomes}

Movement limitation, swelling, pain, QOL, radiological results, and the presence of complications in the patient groups (identified by lesion grade and muscle involvement) were recorded. Subjective assessment (improved, no interval change, or worsened) of movement limitation, pain, and QOL was based on the patient's own description (Table 1), while swelling was assessed by visual inspection. The radiological assessment after sclerotherapy or surgery was done using DUS and MRI, and treatment outcomes were classified as aggravated, partially improved $(<25 \%)$, or improved ( $>50 \%)$. The radiological assessment involved measuring the rate of decrease in the length of the longest axis of the lesion on ultrasonography or MRI. The presence of complications after treatment such as skin necrosis, venous thrombosis, and nerve injury were also evaluated. Additionally, imaging and visual inspection were used to compare outcomes between patients receiving sclerotherapy and those undergoing surgical excision. For patients who underwent sclerotherapy, only the results after single sclerotherapy were shown for postoperative results. 
Table 1. Comparison of clinical outcomes of IMVM treatment by surgery versus sclerotherapy

\begin{tabular}{|c|c|c|c|}
\hline Variable & $\begin{array}{l}\text { Surgical excision } \\
\text { group }(n=36)\end{array}$ & $\begin{array}{l}\text { Sclerotherapy } \\
\text { group }(n=27)\end{array}$ & P-value \\
\hline Pain & & & 0.471 \\
\hline Improved & $18(50)$ & $13(49)$ & \\
\hline No interval change & $17(47)$ & $11(40)$ & \\
\hline Worsened & $1(3)$ & $3(11)$ & \\
\hline Limitation of motion & & & $0.010^{\mathrm{a})}$ \\
\hline Improved & $15(42)$ & $3(11)$ & \\
\hline No interval change & $19(53)$ & $22(81)$ & \\
\hline Worsened & $2(5)$ & $2(7)$ & \\
\hline Swelling & & & 0.322 \\
\hline Improved & $21(58)$ & $17(63)$ & \\
\hline No interval change & $14(39)$ & $7(26)$ & \\
\hline Worsened & $1(3)$ & $3(11)$ & \\
\hline QOL & & & $0.013^{\mathrm{a})}$ \\
\hline Improved & $26(72)$ & $10(37)$ & \\
\hline No interval change & $9(25)$ & $15(56)$ & \\
\hline Worsened & $1(3)$ & $2(7)$ & \\
\hline Radiological outcome & & & $0.017^{\text {a) }}$ \\
\hline Improved & $25(69)$ & $10(37)$ & \\
\hline No interval change & $10(28)$ & $12(33)$ & \\
\hline Worsened & $1(3)$ & $5(19)$ & \\
\hline \multicolumn{4}{|l|}{ Complications } \\
\hline Skin necrosis & 0 & 0 & \\
\hline Vein thrombosis & 0 & 0 & \\
\hline Nerve injury & 0 & 0 & \\
\hline Fibrosis & $1(7)$ & $3(11)$ & 0.206 \\
\hline No complication & $14(93)$ & $24(89)$ & 0.533 \\
\hline
\end{tabular}

Values are presented as number (\%).

IMVM, intramuscular venous malformation; QOL, quality of life.

a) Statistically significant: $\mathrm{P}<0.05$

\section{Statistical analysis}

Statistical analyses were carried out using SPSS version 22.0 (IBM Corp., Armonk, NY, USA). The Fisher exact test was used to compare groups.

\section{RESULTS}

Of the 63 patients who received treatment for IMVM, 36 underwent surgical excision and 27 received sclerotherapy. The surgical excision group exhibited an equal sex distribution (18 male and 18 female patients), while the sclerotherapy group included 12 male and 15 female patients. Patients 20-39 years old were more prevalent in the surgical excision group $(n=17)$, while patients $0-19$ years old were predominant in the sclerotherapy group $(n=13)$. The distribution of IMVM location was as follows: (1) surgical excision group: calf $(\mathrm{n}=11,30 \%)$, thigh $(\mathrm{n}=7,19 \%)$, and head and neck region $(\mathrm{n}=5,14 \%)$; and (2) sclerotherapy group: calf $(\mathrm{n}=8,30 \%)$, head and neck region $(n=8,30 \%)$, and thigh $(n=5,18 \%)$. In both groups, grade 1 lesions were the most prevalent (surgical excision: $n=14,38.89 \%$;
Table 2. Demographics of patients treated for IMVM by surgery or sclerotherapy, including age, sex and characteristics of the IMVM lesion

\begin{tabular}{|c|c|c|c|}
\hline Characteristics & $\begin{array}{l}\text { Surgical excision } \\
\text { group }(n=36)\end{array}$ & $\begin{array}{l}\text { Sclerotherapy } \\
\text { group }(n=27)\end{array}$ & P-value \\
\hline Age (yr) & & & 0.718 \\
\hline $0-19$ & $13(36.1)$ & $13(48.1)$ & \\
\hline $20-39$ & $17(47.2)$ & $9(33.3)$ & \\
\hline $40-59$ & $6(37.5)$ & $5(18.5)$ & \\
\hline$\geq 60$ & 0 & 0 & \\
\hline Sex & & & 0.557 \\
\hline Male & $18(50.0)$ & $15(55.5)$ & \\
\hline Female & $18(50.0)$ & $12(45.5)$ & \\
\hline Upper extremity & & & 0.215 \\
\hline Shoulder & $1(2.7)$ & 0 & \\
\hline Upper arm & $1(2.7)$ & $3(11.1)$ & \\
\hline Forearm & $4(11.1)$ & 0 & \\
\hline Hand & $2(5.5)$ & $1(3.7)$ & \\
\hline Back & $3(8.3)$ & $1(3.7)$ & \\
\hline Lower extremity & & & 0.153 \\
\hline Thigh & $7(19.4)$ & $5(18.5)$ & \\
\hline Calf & $11(30.5)$ & $8(29.6)$ & \\
\hline Foot & $3(8.3)$ & $1(3.7)$ & \\
\hline Head and neck & $5(13.8)$ & $8(29.6)$ & \\
\hline IMVM size & & & 0.147 \\
\hline Grade 1 & $14(38.9)$ & $11(40.8)$ & \\
\hline Grade $2 \mathrm{~A}$ & $7(19.4)$ & $6(22.2)$ & \\
\hline Grade 2B & $8(22.2)$ & $5(18.5)$ & \\
\hline Grade 3 & $7(19.4)$ & $5(18.5)$ & \\
\hline Muscle involvement & & & 0.255 \\
\hline Single muscle & 34 (94.4) & $25(92.6)$ & \\
\hline 2 or more muscles & $2(5.6)$ & $2(7.4)$ & \\
\hline
\end{tabular}

Values are presented as number (\%).

IMVM, intramuscular venous malformation.

sclerotherapy: $\mathrm{n}=11,40.74 \%)$. Single muscle involvement was seen in 34 (94.4\%) and 25 (92.6\%) patients in the surgical excision and sclerotherapy groups, respectively, while multiple muscles were involved in two patients (5.6\%) in the surgical excision group and two patients $(7.4 \%)$ in the sclerotherapy group ( $\mathrm{Ta}-$ ble 2). The IMVM classification was also investigated, and the distribution of patients according to grade (grade 1, grade $2 \mathrm{~A}$, grade $2 \mathrm{~B}$, and grade 3 ), muscle involvement (one muscle or $\geq 2$ muscles), and type of treatment (surgery or sclerotherapy) is shown in Table 3.

Clinical outcomes such as pain, limited movement, swelling, QOL, radiological outcomes, and occurrence of complications were examined and grouped into the categories of improved, no interval change, or worsened. Data showed that, for pain, $13 \mathrm{pa}-$ tients (49\%) improved, 11 patients (40\%) exhibited no interval change, and three patients (11\%) worsened in the sclerotherapy group. In the surgical excision group, 18 patients (50\%) improved, 17 patients (47\%) exhibited no interval change, and one patient (3\%) worsened. However, no statistically significant 
Table 3. Distribution of IMVM patients by grade, muscle involvement, and treatment

\begin{tabular}{|c|c|c|c|c|c|c|c|c|}
\hline \multirow{2}{*}{ Extent of IMVM } & \multicolumn{4}{|c|}{ Single muscle } & \multicolumn{4}{|c|}{2 or more muscles } \\
\hline & Grade 1 & Grade 2A & Grade $2 \mathrm{~B}$ & Grade 3 & Grade 1 & Grade $2 \mathrm{~A}$ & Grade 2B & Grade 3 \\
\hline Sclerotherapy $(n=27)$ & $10(37.0)$ & $6(22.2)$ & $4(14.8)$ & $4(14.8)$ & $1(3.7)$ & 0 & $1(3.7)$ & $1(3.7)$ \\
\hline Surgical excision $(n=36)$ & $13(36.1)$ & $7(19.4)$ & $8(22.2)$ & $7(19.4)$ & $1(2.7)$ & 0 & 0 & 0 \\
\hline
\end{tabular}

Values are presented as number (\%).

IMVM, intramuscular venous malformation.
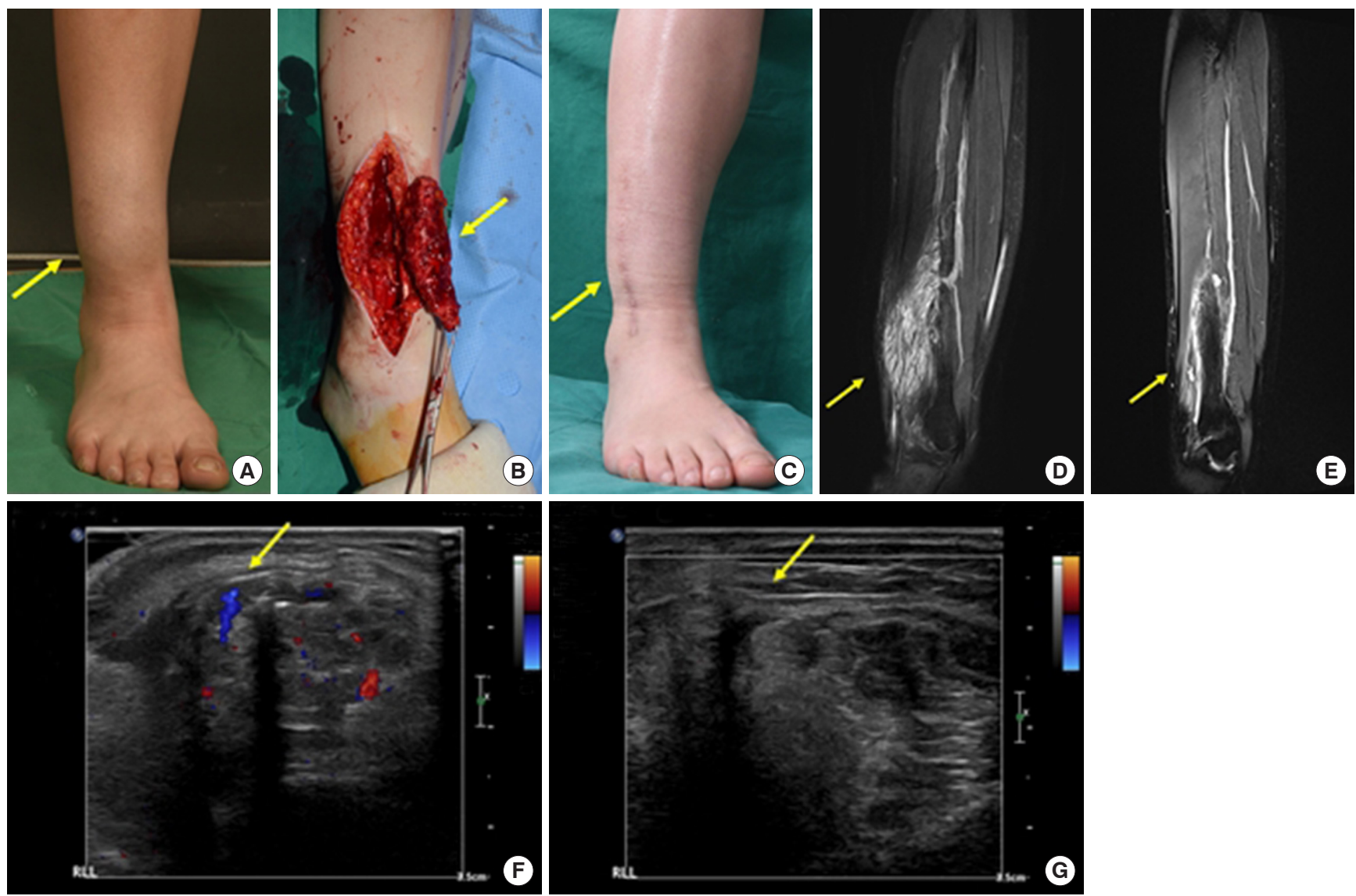

Fig. 1. Surgical excision in a 22-year-old female patient diagnosed with intramuscular venous malformation (IMVM) involving the right ankle muscle (extensor digitorum longus). (A) Preoperative photograph. (B) Intraoperative photograph. (C) Postoperative (2 years) photograph. (D) Preoperative magnetic resonance imaging (MRI) findings (grade 2A). (E) Postoperative (2 years) MRI findings (grade 1). (F) Preoperative duplex ultrasonography (DUS) findings (grade 2A). (G) Postoperative (2 years) DUS findings. Yellow arrow indicates an IMVM lesion.

differences between the groups were observed $(\mathrm{P}=0.471)$. Three patients (11\%) in the sclerotherapy group and 15 patients (42\%) in the surgical excision group exhibited a statistically significant $(\mathrm{P}=0.010)$ improvement. With regard to swelling, 17 patients (63\%) in the sclerotherapy group and $21 \mathrm{pa}$ tients $(58 \%)$ in the surgical excision group exhibited improvement, although this difference was not statistically significant. The QOL significantly improved $(\mathrm{P}=0.013)$ in 10 patients $(37 \%)$ in the sclerotherapy group and in 26 patients $(72 \%)$ in the surgical excision group. A similar pattern $(\mathrm{P}=0.017)$ was observed with regard to radiological outcomes, with 10 patients (37\%) in the sclerotherapy group and 25 patients (69\%) in the surgical excision group exhibiting improvement (Table 1). Examination of photographs, DUS, and MRI examinations showed that IMVM patients who were treated surgically (Figs. $1,2)$ presented better outcomes compared to those who received sclerotherapy (Fig. 3).

\section{DISCUSSION}

The high morbidity and recurrence rates of VMs make the selection of appropriate treatment methods difficult, and this is further complicated by the fact that IMVM lesions typically occur in the deep tissues and are therefore difficult to diagnose by 



Fig. 2. Surgical excision in a 19-year-old male patient diagnosed with intramuscular venous malformation (IMVM) involving the left calf muscle (soleus muscle). (A) Preoperative photograph. (B) Intraoperative photograph. (C) Postoperative (6 months) photograph. (D) Preoperative magnetic resonance imaging (MRI) findings (grade 3). (E) Postoperative (6 months) MRI findings (complete remission). (F) Preoperative duplex ultrasonography (DUS) findings. (G) Postoperative (6 months) DUS findings. Yellow arrow indicates an IMVM lesion.

visual inspection [8]. Therefore, vascular anomalies are typically identified using DUS, and any involvement of surrounding soft tissues and the IMVM lesion size and depth are determined using MRI [9]. Accurate diagnosis of lesions using DUS and MRI helps determine the appropriate treatment protocols.

It is known that IMVMs are benign lesions and do not grow except in children during growth phases [10]. Therefore, treatment methods typically focus on relief of associated symptoms as needed. Although sclerotherapy is less invasive and may be associated with fewer complications, complete removal of the lesion is difficult. In contrast, surgical excision makes complete lesion removal possible, but is more invasive and may be associated with more complications. Therefore, appropriate treatment selection based on informed clinical judgment is very important. In the current study, patients who wanted conservative treatment and did not have any presenting symptoms were reviewed annually and the direction of treatment was chosen based on symptoms and the extent of functional limitations, the purpose 

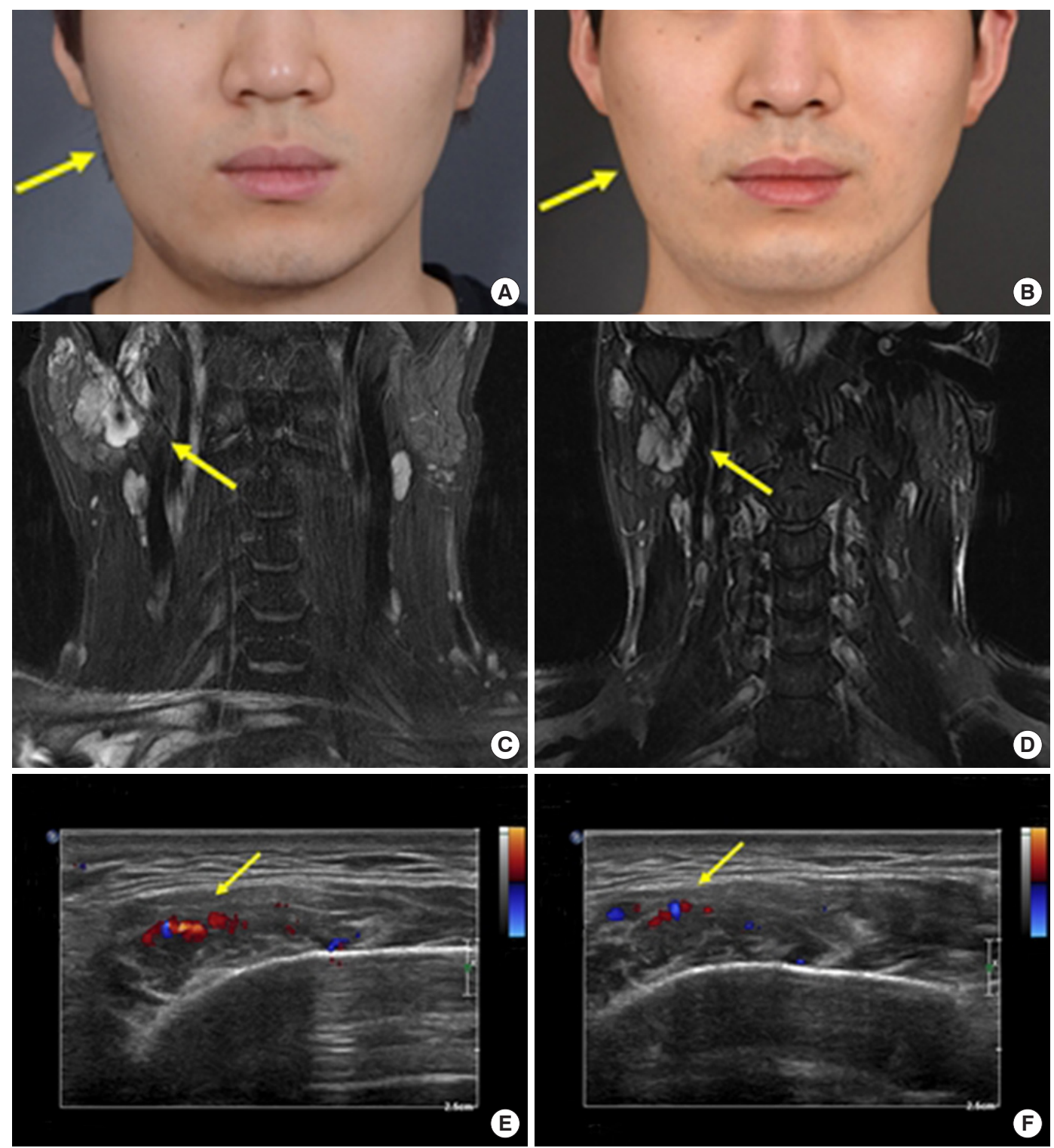

Fig. 3. A 25-year-old male patient received sclerotherapy for intramuscular venous malformation (IMVM) involving the right cheek muscles (masseter, medial pterygoid muscle, lateral pterygoid muscle). (A) Preprocedural photograph. (B) Postprocedural (5 years) photograph. (C) Preprocedural magnetic resonance imaging (MRI) findings (grade 2A). (D) Postoperative (5 years) MRI findings (grade 2A). (E) Preoperative duplex ultrasonography (DUS) findings. (F) Postoperative (4 years) DUS findings. Yellow arrow indicates an IMVM lesion.

of treatment, and patient preferences. Sclerotherapy was used for pain management, while those presenting with functional limitations (microchannel-type localized lesions or severe fibrosis) were treated surgically.

This study compared changes in symptoms, the occurrence of complications, and visual and imaging findings between the sclerotherapy and surgical excision groups. The findings showed a significantly greater improvement in limitation of movement $(\mathrm{P}=0.010)$, $\mathrm{QOL}(\mathrm{P}=0.013)$, and radiological outcomes (DUS and MRI findings; $\mathrm{P}=0.017)$ in the surgical excision group than in the sclerotherapy group (Table 3 ), suggesting that it was a superior treatment option for relieving symptoms and addressing cosmetic discomfort caused by the lesion (Figs. 1-3). No statistically significant differences in complication rates were observed between the two groups.

Previous studies have demonstrated the advantages of sclerotherapy, with Crawford et al. [11] reporting improvement of pain levels in $57.9 \%$ of patients and complete remission in $21.1 \%$ of patients. Ozaki et al. [12] found that eight out of 10 patients in their study experienced improvement in pain levels and two patients exhibited complete pain alleviation with sclerotherapy, while Bianchini et al. [13] reported pain improvement in $88.9 \%$ of patients included in their cohort. Therefore, it was concluded that sclerotherapy should be performed as the 
first of line treatment for general VM, with surgical excision being carried out in case of sclerotherapy failure $[14,15]$.

However, the results of the current study showed that surgical excision was effective in improving symptoms of IMVM, suggesting that sclerotherapy should be considered in situations where the patient refuses surgery, the goal of treatment is pain improvement and not complete remission, imaging shows no evidence of microchannel lesions or severe fibrosis, and surgical excision would likely result in a large scar due to the lesion size. The results of this study suggest that surgical excision is the most effective treatment option for IMVMs unless contraindicated. Although several studies have examined IMVM treatment methods, no clear guidelines for treatment selection have been developed. Based on the results of this study, surgical excision is encouraged for the treatment of IMVMs.

\section{NOTES}

\section{Conflict of interest}

Ho Yun Chung is an editorial board member of the journal but was not involved in the peer reviewer selection, evaluation, or decision process of this article. No other potential conflicts of interest relevant to this article were reported.

\section{Ethical approval}

The study was approved by the Institutional Review Board of Kyungpook National University Hospital (IRB No. 2018-10010) and performed in accordance with the principles of the Declaration of Helsinki. The informed consent was waived because this study design is a retrospective chart review.

\section{Patient consent}

The patients provided written informed consent for the publication and the use of their images.

\section{Author contribution}

Conceptualization: HY Chung. Data curation: YH Kim, JS Lee, JM Lee, JY Kim. Formal analysis: YH Kim, JY Ryu. Funding acquisition: SJ Lee. Methodology: YH Kim, JS Lee, SJ Lee. Project administration: YH Kim, S Huh, JY Kim. Visualization: YH Kim, JM Lee, SY Lee, S Huh. Writing - original draft: YH Kim. Writing - review \& editing: HY Chung. All authors read and approved the final manuscript.

\section{ORCID}

Yun Hyun Kim

Jeong Yeop Ryu

Joon Seok Lee https://orcid.org/0000-0002-9137-0909

https://orcid.org/0000-0003-2812-5051

https://orcid.org/0000-0002-1580-0487
Seok Jong Lee

Jong Min Lee

Sang Yub Lee

Seung Huh

Ji Yoon Kim

Ho Yun Chung
https://orcid.org/0000-0002-6131-632X

https://orcid.org/0000-0002-4163-913X

https://orcid.org/0000-0001-8529-8229

https://orcid.org/0000-0002-0275-4960

https://orcid.org/0000-0001-7998-8936

https://orcid.org/0000-0001-7359-3044

\section{REFERENCES}

1. Carabin J, Bouhamama A, Vaz G, et al. Percutaneous cryoablation of symptomatic intramuscular venous malformation. J Vasc Interv Radiol 2020;31:558-63.

2. International Society for the Study of Vascular Anomalies (ISSVA). Classification [Internet]. Milwaukee, WI: ISSVA; c2018 [cited 2021 May 15]. Available from: https://www. issva.org/classification.

3. Vogel SA, Hess CP, Dowd CF, et al. Early versus later presentations of venous malformations: where and why? Pediatr Dermatol 2013;30:534-40.

4. Hein KD, Mulliken JB, Kozakewich HP, et al. Venous malformations of skeletal muscle. Plast Reconstr Surg 2002; 110:1625-35.

5. Rivas S, Lopez-Gutierrez JC, Diaz M, et al. Venous malformations: diagnosis and treatment during the childhood. Cir Pediatr 2006;19:77-80.

6. Jung HC, Kim DH, Park BK, et al. Extensive intramuscular venous malformation in the lower extremity. Ann Rehabil Med 2012;36:893-6.

7. Domb BG, Khanna AJ, Mitchell SE, et al. Toe-walking attributable to venous malformation of the calf muscle. Clin Orthop Relat Res 2004;(420):225-9.

8. Goyal M, Causer PA, Armstrong D. Venous vascular malformations in pediatric patients: comparison of results of alcohol sclerotherapy with proposed MR imaging classification. Radiology 2002;223:639-44.

9. Laurian C, Cerceau P, Paraskevas N, et al. Intramuscular venous malformations of the calf: surgical treatment outcomes of 57 patients. Phlebology 2020;35:597-604.

10. Park H, Kim JS, Park H, et al. Venous malformations of the head and neck: a retrospective review of 82 cases. Arch Plast Surg 2019;46:23-33.

11. Crawford EA, Slotcavage RL, King JJ, et al. Ethanol sclerotherapy reduces pain in symptomatic musculoskeletal hemangiomas. Clin Orthop Relat Res 2009;467:2955-61.

12. Ozaki M, Kurita M, Kaji N, et al. Efficacy and evaluation of safety of sclerosants for intramuscular venous malformations: clinical and experimental studies. Scand J Plast Reconstr Surg Hand Surg 2010;44:75-87. 
13. Bianchini G, Camilli D, Furgiuele S. Intramuscular venous malformations of the upper and lower limbs: indications and outcomes of sclerotherapy. Cardiovasc Intervent Radiol 2018;41:1505-12.

14. Ryu JY, Lee JS, Lee JW, et al. Clinical approaches to vascular anomalies of the lip. Arch Plast Surg 2015;42:709-15.

15. Hassanein AH, Mulliken JB, Fishman SJ, et al. Venous malformation: risk of progression during childhood and adolescence. Ann Plast Surg 2012;68:198-201. 